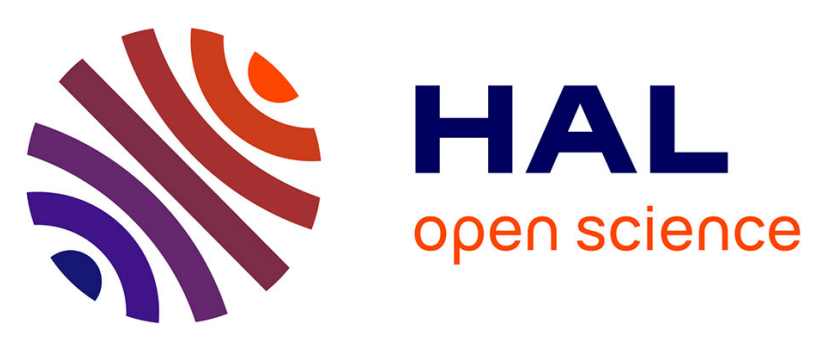

\title{
A didactic-based model of scenarios for designing an adaptive and context-Aware Learning System
}

\author{
Jean-Louis Tetchueng, Serge Garlatti, Sylvain Laubé
}

\section{To cite this version:}

Jean-Louis Tetchueng, Serge Garlatti, Sylvain Laubé. A didactic-based model of scenarios for designing an adaptive and context-Aware Learning System. IEEE/WIC/ACM : International Conference on Web Intelligence, November 2 - 5, Silicon Valley, USA, Nov 2007, Silicon Valley, United States. hal-02142969

\section{HAL Id: hal-02142969 \\ https://hal.science/hal-02142969}

Submitted on 29 May 2019

HAL is a multi-disciplinary open access archive for the deposit and dissemination of scientific research documents, whether they are published or not. The documents may come from teaching and research institutions in France or abroad, or from public or private research centers.
L'archive ouverte pluridisciplinaire HAL, est destinée au dépôt et à la diffusion de documents scientifiques de niveau recherche, publiés ou non, émanant des établissements d'enseignement et de recherche français ou étrangers, des laboratoires publics ou privés. 


\title{
A Didactic-based Model of Scenarios for designing an Adaptive and Context-Aware Learning System
}

\author{
Jean-Louis Tetchueng ${ }^{1}$, Serge Garlatti ${ }^{1}$, Sylvain Laube ${ }^{2}$ \\ ${ }^{1}$ ENST Bretagne, GET CS 83818, 29238 Brest Cedex, France \\ \{jl.tetchueng,serge.garlatti\}@enst-bretagne.fr \\ ${ }^{2}$ CREAD, IUFM Bretagne, 8, rue d'Avranches, 29200 Brest, France \\ sylvain.laube@bretagne.iufm.fr
}

\begin{abstract}
Nowadays, technology-enhanced learning systems must have the ability to reuse learning resources from distributed repositories, to take into account the context and to allow dynamic adaptation to different learners based on substantial advances in pedagogical theories and knowledge models. We focus on learning systems using a problem-based learning approach represented by scenarios. In our framework, the goal of scenarios is to describe the learning and tutoring activities to acquire some knowledge domain (for instance physics) and knowhow to solve a particular problem. The main issue is to design a generic scenario which can deal with most of learning situations. From a generic scenario, the $e$ learning system will compute on the fly a particular scenario dedicated to the current learner and its learning situation. The main contribution of this paper is a semantic and didactic-based model of scenarios for designing an adaptive and context-aware learning System. The scenario model is acquired from: i) the know-how and real practices of teachers ii) the theory in didactic anthropology of knowledge of Chevallard [1]; iii) a hierarchical task model.
\end{abstract}

\section{Introduction}

Nowadays, technology-enhanced learning systems must have the ability to reuse learning resources (learning objects, tools and services) from large repositories, to take into account the context and to allow dynamic adaptation to different learners based on substantial advances in pedagogical theories and knowledge models [2]. Thus, the design and engineering of learning systems must be considered as an interdisciplinary problem requiring the integration of different scientific approaches from computer science, pedagogical and/or didactical theories, education, etc. The design process leads to an artifact - the learning system - based on different scientific approaches which are related to different theories - for instance, activity theory, theory of didactic situations, computerbased theories, etc. Consequently, it is crucial to establish the relationships between theories, models and artifacts to ensure the traceability and the interpretation of phenomena related to the use of artifacts [3].

Adaptive technology-enhanced learning systems compute on the fly the delivered courses from distributed data resources, according to the current context and the learner's needs. The resource reusability has to rely on resource interoperability at syntactic and semantic level. At semantic level, resources are described by semantic metadata and their corresponding ontologies. These ontologies are used to formalize at knowledge level the different required models of learning systems: learner and teacher models, domain model, context model, scenario models, pedagogical and/or didactical models, adaptation models and rules, etc. New software architectures are necessary to use learning system models based on ontologies and to support dynamic adaptation and context awareness. We designed a flexible and adaptive composition engine, called SCARCE - SemantiC and Adaptive Retrieval and Composition Engine - to design such technology-enhanced learning systems [4].

We are interested in technology-enhanced learning systems using a problem-based learning approach, represented by scenarios. In our framework, the goal of scenarios is to describe the learning and tutoring activities to acquire some knowledge domain (for instance physics) and know-how to solve a particular problem. A scenario may depend on several dimensions which describe different learning situations (in some way): the learning domain (course topic), the learner (his know-how and knowledge levels), the tutor/teacher, the learning and tutoring activities (their typology, organization and coordination), the activity distribution among learners, 
teachers and computers, the learning "procedures" according to a particular school/institution/university and the didactical / pedagogical environment. Research on the learning scenario models leads to the standardization of pedagogical approaches - for instance IMS LD [5]. These models enable authors/teachers to produce generic and standard models which are neutral on a pedagogical and/or didactical point of view [6]. In other words, there are unable to deal with all mentioned dimensions.

The main contribution of this paper is a semantic and didactic-based model of scenarios which will be used in the flexible composition engine SCARCE for designing an adaptive and context-aware learning System. The different scenario dimensions are acquired from: i) the know-how and real practices of teachers in a problem-based learning approach in a particular framework: an institution IUFM, different categories of probationary teachers, a course about "the air as gas in its static and dynamic aspects: properties, theory and applications"; ii) the theory in didactic anthropology of knowledge of Chevallard [1]; iii) a hierarchical task model. A co-design methodology has been used to articulate teacher real practices, the Chevallard theory and the hierarchical task model to define the different learning system models [7]. The hierarchical task model enables us to define the learning and tutoring activities, the activity distribution among learners, teachers and computers and also to transpose the main concepts of the Chevallard theory. Adaptation policies lean on teacher real practices and the Chevallard theory. The didactical environment acquired from the Chevallard theory and the teacher real practices and know-how is managed by a context model.

First of all, we briefly present the main features of the SCARCE environment and more particularly, how our scenario model will be used in the environment. Secondly, we present the outcome of the co-design methodology for acquiring the problem-based learning scenarios from teacher real practices and the Chevallard theory in didactic anthropology of knowledge. Thirdly, the computer-based model of scenarios is detailed. The transposition of the main concepts of Chevallard theory and the typology of learning and tutoring activities is explained. Finally, the conclusion highlights the main results of this study. We also point out the next research issues.

\section{The SCARCE Environment}

The adaptive learning system can be viewed as an adaptive virtual document. It will use a flexible composition engine, called SCARCE - SemantiC and Adaptive Retrieval and Composition Engine based on a semantic web approach [4]. SCARCE is the core of
ICCARS $^{1}, \mathrm{CANDLE}^{2}$ and $\mathrm{KMP}^{3}$ projects. In our framework - the MODALES project (Modeling Didacticbased Active Learning Environment in Sciences)-, a learning system consists of a set of resources, their metadata and the corresponding ontologies and an adaptive composition engine which is able to select the relevant resources, to organize and to assemble them by adapting the delivered course to the learner needs and the current learning situation. To provide flexibility, selection, organization and adaptation are parameters of the composition engine and lead to a specification.

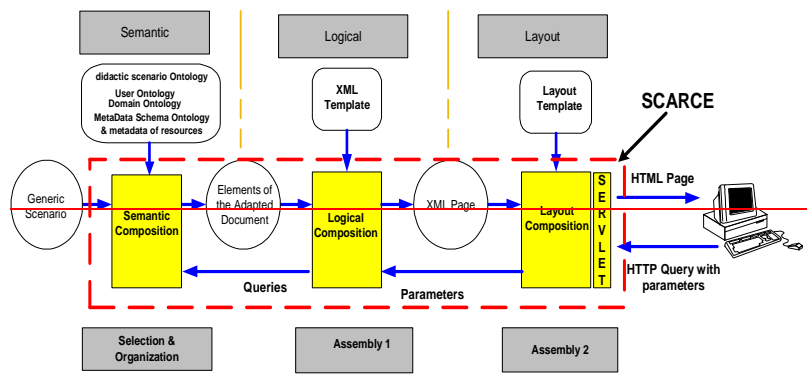

Figure 1. The composition engine architecture

In Figure 1, the composition engine uses four loosely coupled ontologies which are: metadata ontology at the information level which describes the indexing structure of resources, some index values are taken in the domain and scenario ontologies; domain ontology representing knowledge in a specific area - physics, didactic, epistemology; scenario ontology consisting of a scenario model - organization and selection - and an adaptation model. The scenario model defines the core concepts of the organization which is a directed graph. Thus, it defines the different types of nodes and links and the different sub-types of nodes and links which depend on the application. In our framework, it is respectively the main concepts of a hierarchical task model and the didactical concepts and their features. The scenario and adaptation model leads to a scenario ontology based on a hierarchical task model and an adaptation model based on the adaptation policies required by the MODALES Project; a learner and teacher ontology which defines different stereotypes - categories of probationary teachers and teachers - and individual features. Metadata schema, ontologies and specifications are based on the explicit common knowledge shared by all community members. In other words, scenarios are key issue to design the scenario

${ }^{1}$ ICCARS : Integrated and Collaborative Computer Assisted Reporting System

${ }^{2}$ CANDLE : Collaborative And Network Distributed Learning Environment

${ }^{3}$ KMP: Knowledge Management Portal, RNRT Project 
ontology, adaptation ontology and specifications. At present, SCARCE is not context-aware. It does not manage a context ontology - formalizing a context model.

The specification is called a generic scenario (see Figure 1). It has to deal with most of learning situations. From a generic scenario, the learning system will compute on the fly a specific scenario dedicated to the current learner and his/her learning situation. The generic scenario acts as scaffolding in the learning system. Acquiring the scenario model is not an easy task because it is necessary to make explicit knowledge, know-how and real practices of teachers and to establish the relationships between theories, models and artifacts. After a short introduction of the MODALES Project, the outcome of the acquisition of teacher practices and know-how is presented.

\section{The MODALES Project}

The MODALES project is aimed at designing an adaptive learning system for probationary teachers, based on real practices and teacher know-how. The course topic is about "the air as gas in its static and dynamic aspects: properties, theory and applications" for different categories of probationary teachers - called learners. The learners are probationary teachers: primary school teachers (called PE for "professeur des Ecoles" and secondary school teachers (called PLC for "professeur des Lycées et Collèges": earth/biology sciences and physic. The teachers are considered as experts in education. The course topic is about "the air as gas in its static and dynamic aspects: properties, theory and applications" for different categories of probationary teachers. In our framework, scenarios may change according to the following features: i) the category of learners having intra and inter category variability; ii) the available resources from different didactical environment and domains physics, didactic and epistemology - which can be determined by teachers iii) distance or face-to-face activity according to learner needs, learning policy and didactical environment constraints iv) the sharing of activities between teachers, learners and computers according to learner needs and learning policies. These features will lead to adaptation policies according to the current learner and context in the learning system. The main issue is to design a generic scenario which can deal with most of learning situations (from a computer science viewpoint).

\section{Acquisition of teacher practices and know- how}

Firstly, several scenarios based on a common learning scenario $\mathrm{P}_{\mathrm{o}}$ (whose variables are learners, the expert teacher and the available resources) were built [8]. Each scenario is composed of two phases: 1) construction of professional references for teaching, 2) development of a training sequence implemented in classrooms. Different features are associated to activities in the two phases: 1) activation of the phase (if it exists in the scenario); 2) distance or face to face; 3 ) a description of the available resources and their domain (physics, didactic, epistemology, history); 4) an activity description for learners and teachers.

Secondly, we use the theory in didactic anthropology of knowledge of Chevallard to go further [8]. The praxeology system $(T / \tau / \theta / \Theta)$ of the Chevallard theory enables us to acquire the scenario model and the didactical environment. According to Chevallard, teacher and learner activities can be described in terms of types of tasks Tc achieved by techniques $\tau$ which may be recursively achieved by subtasks Tc'. Thus, a Task/Technique system $(T / \tau)$ has a hierarchical structure. This hierarchical structure $(T / \tau)$ defines a know-how that leans on an environment composed of a technology $\theta$ (discourse that justifies and explains techniques) and a theory $\Theta$ justifying and highlighting the technology. In other words, a Task/Technique system $(T / \tau)$ describes a type of problem $(T)$ to solve and the technique $(\tau)$ describes how to solve it $(T)$.

We can observe six different moments in the didactical organization [1]: i) the first encounter with the type of tasks Tc (M1); ii) the exploration of the type of tasks Tc and the construction of techniques $\tau$ (M2); iii) the technique work that improves the technique and makes it more efficient (M3); iv); the construction of a Technology/Theory related to technique $\tau$ (M4) v) the institutionalization of the system $(T / \tau / \theta / \Theta)$ by the teacher (M5); vi) the evaluation (M6) (cf. Figure 2). For a given technique, a task can be decomposed into sub-tasks which are achieved according to specified operators. At present, three different operators are used: sequence ('seq'), alternative ('alt') and parallel ('par').

Moreover, the scenario analysis shows different categories of learning and tutoring tasks, organized at different levels of the task hierarchy: scenario, phase, moment, learning task, routine task and tutoring task as shown in the figure 2. Two categories of Task/Technique systems can also be observed according to the teacher role: routine and problematic system. In the former, the teacher did not plan to intervene because the learner is able to solve the problem. In the latter, more the teacher 
intervenes quickly in the learning situation, more the Task/Technique system is considered as problematic. In this paper we focus on the problematic systems. The activity structure and distribution among learners, teachers and computers depend on these two types of Task/Technique system $(T / \tau)$. Nevertheless, we may have different problematic systems according to the different learning situations.

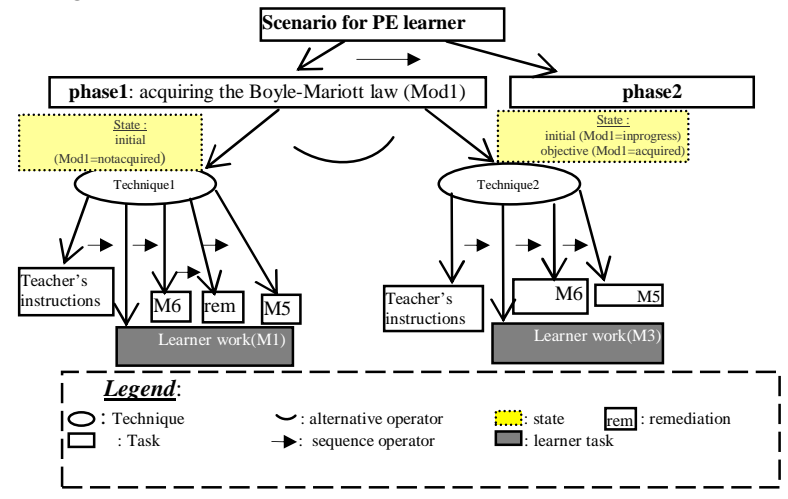

Figure 2. A partial scenario having two alternative techniques in the "phase1" task for a PE learner.

The adaptation of scenarios leads to choose the relevant technique according to the learners and the didactical environment. According to the Task/Technique system, the choice can be done by the computer, the learner or the teacher. The selection of the relevant technique depends on the following properties: the Task/Technique systems, the learner category (PE, PLC, type of PLC, etc.), the learner curriculum and the didactical environment. From the Chevallard theory and the teacher real practices and know-how, we define the didactical environment as follows: type of classrooms (virtual classroom, scientific laboratory with or without computers and/or with or without internet access, associated CITT $_{4}$ tools (chat, email, forum, etc.), technical instruments (thermometer, barometer, etc.), resources (documents, experiments, etc.) and face to face or at distance.

First of all, we explain how the learner and the technique properties are used to choose the relevant technique in a particular didactical environment. Secondly, we detail the different roles of the didactical environment features.

To illustrate the Chevallard's theory and its concepts, we choose a particular case study for a PE learner in which we detail the task "phase 1" achieved with two different techniques which correspond to a problematic task. We assume that these techniques are relevant for the current learner and the current didactical environment.

4 Communication and Information Technologies for Teaching
The course topic is about "the air as gas in its static and dynamic aspects: properties, theory and applications". In the Chevallard framework, the considered theory is thermodynamics. In physics, theories can be "evaluated" by means of different laws. In our case, it is the BoyleMariotte law which is represented as follows $(\mathrm{PV} / \mathrm{T}=\mathrm{K})$ for PE Learners and $(\mathrm{PV} / \mathrm{T}=\mathrm{nRT})$ for PLC learners. The knowledge domain is composed of the thermodynamic theory, the corresponding laws $(\mathrm{PV} / \mathrm{T}=\mathrm{K})$, the related concepts (Pressure P, Volume V, Temperature T) and their relationships. By means of a particular Task/Technique system $(\mathrm{T} / \tau)$, a PE learner may learn some knowledge about the thermodynamic theory, the $(\mathrm{PV} / \mathrm{T}=\mathrm{K})$ law, the related $\mathrm{P}, \mathrm{V}$ and $\mathrm{T}$ concepts and some know-how: how to measure a pressure, how to measure a temperature, how to demonstrate the $(\mathrm{PV} / \mathrm{T}=\mathrm{K})$ law by using a barometer and a thermometer. To deal with the learner know-how and knowledge levels, the knowledge domain entities (theories, laws, concepts and relationships) and the type of tasks may have three different states: "not acquired", "in progress", "acquired". For a given task, the state "not acquired", correspond to the moment M1 and the states "in progress" and "acquired" correspond to the moment M2 and M3. After an evaluation task, a teacher or the computer can update the learner know-how and knowledge levels for some domain entities and for the current task, for instance from "in progress" to "acquired" if the know-how of the current task is considered as acquired.

In Figure 2, the two techniques are annotated with the knowledge and know-how levels: the prerequisite and outcome states of the learner. When it is the first encounter of the task "phase 1", the corresponding learner state is "not acquired". Thus, the relevant technique is "technique 1". After a successful evaluation sub-task, his outcome state will be "in progress" for the task. If the learner state for the task "phase 1" is "in progress", the relevant technique is "technique 2". After a successful evaluation sub-task, his outcome state will be "acquired" for the task. Thus, the adaptation of scenarios leads to choose the relevant technique according to the learner and the current didactical environment. Several techniques are normally associated to the task "phase 1 " to deal with the different categories of learners and the different didactical environments. Whether the learner state for the concept "P", "V" and "T" are "not acquired" or "in progress", the relevant technique must have the corresponding prerequisite states and must consist of sub-tasks dedicated to the acquisition of the corresponding knowledge.

From the didactical environment, we firstly explain the role of the technical instruments. An historical and epistemological analysis of several historical and didactical situations shows that laws in physics are tested by means of technical instruments [9]; For instance, the 
technical instruments could be a thermometer and a barometer. Thus, the learners must have or acquire knowhow to use these technical instruments to solve the problem related to the task "phase 1". Whether the learner state for these tasks "temperature and pressure measurements" are "not acquired" or "in progress", the relevant technique must have the corresponding prerequisite states and must consist of sub-tasks dedicated to the acquisition of the corresponding know-how.

The "face to face" or "at distance" feature change the Task/Technique system and the activity distribution among learners, teachers and computers. It is the same for the type of classrooms and the CITT tools. Moreover, some specific know-how may be assumed (internet access and information gathering, forum, chat, etc.) to achieve communication tasks or information retrieval tasks. Thus, such know-how must be routine tasks or at least acquired. Otherwise, it is necessary to have sub-tasks to acquire such know-how.

In conclusion, we showed that, it is necessary to describe the different techniques according to the learner and the didactical environment features to be able to choose the relevant technique.

\section{A didactic based model of scenario}

From the acquisition of teacher real practices by means of the Chevallard theory, the didactic-based scenario model is transposed into a computer-based hierarchical task model. Firstly, we describe and justify the transposition of the Task/Technique systems and their hierarchical structure. Secondly, we analyze the representation of the typology of learning and tutoring activities. Finally, we show how the adaptation is formalized according to parameters describing the learner, the context.

\subsection{The task/technique system transposition}

Teaching and learning activities of scenarios have been described in terms of type of tasks $T_{c}$ and techniques $\tau$. The type of tasks $T_{c}$ describes the teaching and learning activities, while techniques $\tau$ describe a way of achieving the type of task $T_{c}$. We transpose the resulting Task/Technique system $\left(\mathrm{T}_{\mathrm{c}} / \tau\right)$ in the task/method paradigm of the hierarchical task model. Therefore, we can represent in this model, the Task/Technique system $\left(\mathrm{T}_{\mathrm{c}} / \tau\right)$ of Chevallard [1] fitted with its hierarchical structure and didactics properties describing scenarios while we preserve its initial properties and semantics.

Several research studies in artificial intelligence focus on the hierarchical task model using the task/method paradigm [10-13]. In learning environment, hierarchical task models were also used for designing, for instance, authoring tools [14], learning systems [15, 16] and recommender systems [17]. The mechanism of hierarchical and recursive decomposition of a problem into sub-problems is one of the basic characteristics of the hierarchical task model [10-13]. The hierarchical task model consists of abstract and atomic tasks and methods. In a particular task, a method represents the various ways of achieving this task. A method describes the decomposition of its task into sub-tasks. The execution of these sub-tasks is done through a control structure which is composing of the following operators: sequence, parallel, choice. Their respective specifications are quite the same as those of 'seq', 'par' and 'alt' presented in the paragraph 4. Thus, an abstract task can be broken down into abstract or atomic sub tasks through its associated methods. An atomic task is not composed of sub-tasks. It can be achieved by a simple procedure - for instance, an information retrieval process, a particular human computer interaction, etc. Hence, in a computer-based model, the task/method paradigm has respectively a semantic and a hierarchical structure similar to those of the Task/Technique systems $\left(\mathrm{T}_{\mathrm{c}} / \tau\right)$ of Chevallard. Moreover, we have to refine (specialization) the task and method concepts of our model to take into account adaptation and sharing of activities.

\subsection{Transposition of the task type typology}

The typology of tasks of our computer-based model identifies the various types of tasks Tc which compose the scenarios described and represented in paragraph 4: scenario $^{5}$, phase, moment, learning tasks, routine tasks, tutoring tasks.

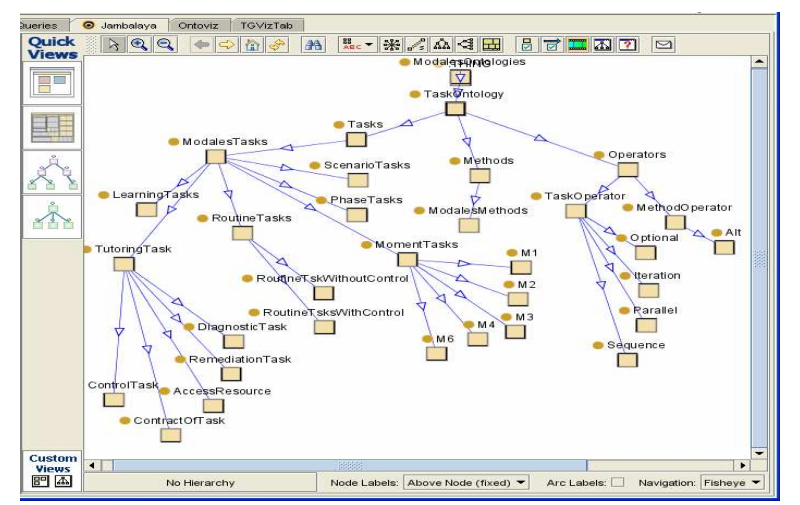

Figure 3. Typology of tasks of the computer-based model.

\footnotetext{
${ }^{5}$ In this paragraph, italic and bold terms represent the concepts of the Chevallard's theory described at the second stage.
} 
One of the main criteria of the formalization of tasks represented in figure 3 is their atomic character or not respectively abstract or not. The tasks «ScenarioTasks», «PhaseTasks», «MomentTasks» are represented by abstract tasks since a scenario consists of two phases which are broken down into moments while each moment consists of learning tasks, routine tasks, and/or tutoring tasks. Tasks «LearningTasks» are also represented as abstract tasks, because they represent a Task/Technique system which can be broken down into others sub Task/Technique systems. On the other hand, the task «RoutineTasks» without control, called «RoutineTskWithoutControl», is only composed of atomic tasks. The tasks «TutoringTask» are atomic tasks. They correspond to tutoring activities of the teacher or of the computer system. In both cases, these tasks are seen as "simple procedures".

\subsection{Adaptation of scenarios}

From the Chevallard theory viewpoint, the relevant technique must be selected according to the current learner and the didactical environment. From a computerbased viewpoint, the adaptation process can be viewed as the selection of the relevant method which represents the Chevallard concept of techniques. It aims at a dynamic selection of the relevant methods according to the context and the current learner. The know-how and knowledge levels of the learner are represented by an overlay model [18] associated to the learner model as described in the paragraph 4 (the states associated with the knowledge domain entities).

The context model represents the didactic environment as described in the paragraph 4 . This model will lead to a context ontology in the SCARCE environment. It is described by the type of classroom in which the learning activities will take place, the associated CITT $_{6}$ tools and devices, a list of technical instruments which are a subset of those in the domain, "face-to-face" or "at distance". In figure 2, the context is composed of: a thermometer and a barometer as technical instruments. This scenario is achieved at distance in a laboratory of sciences. The learner is described by his curriculum, his category (PE, PLC, type of PLC, etc.) and his overlay model, that is to say a set of states for some knowledge domain entities (theories, laws and concepts) and for some tasks. These states are assigned to the learner and will be updated. The domain model consists of the thermodynamic theory, the corresponding law $(\mathrm{PV} / \mathrm{T}=\mathrm{K})$, the related concepts (Pressure $\mathrm{P}$, Volume V, Temperature $\mathrm{T}$ ) and their relationships.

6 Communication and Information Technologies for Teaching
The context, learner and domain models will be represented by means of ontologies within SCARCE (SemantiC and Adaptive Retrieval and Composition Engine) environment [4]; SCARCE is a flexible and adaptive composition engine to design E-learning systems in which the scenario model will be implemented. The adaptation process in SCARCE consists of two stages: firstly, resources are evaluated and classified in one equivalence class according to class membership rules. There are up to five equivalence classes of resources for usability reasons, named Very Bad, Bad, To Consider, Good and Very Good - indeed, it might be difficult for a user to deal with too many equivalence classes ; secondly, one adaptation technique is chosen for the current user (annotation, hiding, sorting, direct guidance, etc.). According to this adaptation strategy, some equivalence classes and their resources are selected for the current learner. In the scenario model, we have three adaptation categories: selection of relevant documents (category 1), selection of relevant methods for remediation and evaluation task (category 2) and selection of relevant methods for other learning task (category 3 ). In this paper, we only focus on the latter category (3). At present, we only need two equivalence classes (Good and Bad) for methods of learning tasks in a scenario. In other words, we have to select the "good" methods and to hide the others.

Thus, let $\mathrm{T}_{\mathrm{a}}$ be a task (the task "phase1" on Figure2. for example), $\mathrm{Ci}$ be a context, $\mathrm{A}_{\mathrm{pp}}$ be a learner, $\mathrm{S}_{\text {app }}$ the state of $\mathrm{A}_{\mathrm{pp}}$ compared to the task $\mathrm{T}_{\mathrm{a}}$ and domain entities $((\mathrm{PV} / \mathrm{T}=\mathrm{K}), \mathrm{P}, \mathrm{T}, \mathrm{V}$, thermometer, barometer $)$. The adaptation process is described in three steps, method retrieval, overlay model management and context management as follows:

1) Method retrieval: For the given task $T_{a}$, all corresponding methods $\mathrm{Mi}$ are retrieved. Thus for "phase1", method1 (for technique1) and method2 (for technique2) are retrieved.

2) Overlay model management: If $T_{a}$ belongs to the task set of $\mathrm{A}_{\mathrm{pp}}$, each method Mi for which the prerequisite match up to the corresponding states in $S_{\text {app }}$ is selected. If $\mathrm{T}_{\mathrm{a}}$ not belongs to the task set of $\mathrm{A}_{\mathrm{pp}}$, it is added to the task set of $A_{p p}$ with the state "not acquired". If the set of selected methods is empty, all retrieved methods belong to the equivalence class "Bad". This case is considered as a problematic situation and required a teacher action to remediate or to provide a new method and context adapted to the learner and the task $\mathrm{T}_{\mathrm{a}}$.

3) Context management: the previous selected methods for which the "context" properties match up to the context properties are kept. We can distinguish several cases: (i) If the set of kept methods is empty, it indicates that all methods belong to the equivalence class "Bad". This case 
is also considered as a problematic situation and required a teacher action to remediate or to indicate a new method and context adapted to the learner and the task $\mathrm{T}_{\mathrm{a}}$; (ii) If the set of kept methods is composed of a single method, it belongs to the class "Good" and is proposed to the learner as the relevant one for the resolution of $\mathrm{T}_{\mathrm{a}}$; (iii) If the set of kept methods is composed of several methods; they belong to the equivalent class "Good". In this case, several constraints could be used to filter the set of methods. These constraints can be defined by the teacher. Nevertheless, if there are no constraints, all methods are proposed to $\mathrm{A}_{\mathrm{pp}}$.

\section{Conclusion}

Technology-enhanced learning systems have to reuse learning resources (learning objects, tools and services) from large repositories, to take into account the context and to allow dynamic adaptation to different learners based on substantial advances in pedagogical theories and knowledge models. Thus, we proposed an adaptive and context-aware model of scenarios based on the theory in didactic anthropology of knowledge, the teacher real practices and know-how and a hierarchical task model. The latter enables us to define the learning and tutoring activities, the activity distribution among learners, teachers and computers and also to transpose the main concepts of the Chevallard theory. The context model implements the didactical environment acquired from the Chevallard theory and the teacher real practices and know-how.

Nevertheless, the model is not finished. At present, we only manage one category of adaptation. In other word, we need to continue the co-design process in order to precise the other adaptation categories and to refine the different models. We are developing the hierarchical task model, the typology of tasks in the SCARCE environment and the management of context. Experiments have to be done to evaluate scenarios and adaptive policies afterwards.

\section{Acknowledgments}

The project MODALES receives funding from Brittany region as a PRIR project, and belongs to the ACI GUPTEN Project.

\section{References}

[1] Y. Chevallard, "L'analyse des pratiques enseignantes en théorie anthropologique du didactique," La Pensée sauvage, Grenoble, Recherches en didactique des mathématiques, vol. 19, pp. 221-226, 1999.
[2] N. Balacheff, "10 issues to think about the future of research on TEL," Les Cahiers Leibniz, Kaleidoscope Research Report, 2006.

[3] P. Tchounikine and Al., "Platon-1: quelques dimensions pour l'analyse des travaux de recherche en conception d'EIAH.," Département STIC, CNRS 2004.

[4] S. Garlatti, S. Iksal, and P. Tanguy, "SCARCE: an Adptive Hypermedia Environment Based on Virtual Documents and Semantic Web," in Adaptable and Adaptive Hypermedia Systems, S. Y. Chen and G. D. Magoulas., Eds.: Idea Group Inc., 2004, pp. 206-224.

[5] IMS, "IMS Learning Design Information Model, IMS Global Learning Consortium," 2003.

[6] T. Nodenot, "Etude du potentiel du langage IMS-LD pour scénariser des situations d'apprentissage : résultats et propositions," in Pernin J-P. et Godinet H. (dir.) , actes électroniques du colloque Scénarios, p. 57-63, 2006.

[7] S. Garlatti, J.-L. Tetchueng, S. Laubé, Y. Kuster, O. K. Zein, and Y. Kermarrec, "The Co-Design of Scenarios for a Didacticbased E-learning System viewed as an Adaptive Virtual Document," presented at Satellite Workshop E-Learning, 2nd IEEE International Conference on Information \& Communication Technologies : from Theory to Applications, Damascus, Syria, 2006.

[8] S. Laubé, S. Garlatti, and Al, "Scénarios intégrant les TICE : les méthodologies et les cadres théoriques à l'œuvre dans la recherche MODALES," presented at 8ème Biennale de l'Education, Colloque "Scénariser l'enseignement et l'apprentissage : une nouvelle compétence pour le praticien ?" INRP - ERTé E-Praxis, Lyon, 2006.

[9] M. Guedj, S. Laubé, and P. Savaton, "De l'analyse historique au profit de l'analyse des situations didactiques," Numéro spécial des Cahiers du Centre F. Viète, ouvrage collectif, ReForEHST Ed. (à paraître), 2007.

[10] Pierret-Golbreich, "TASK: un environnement pour le développement de systèmes à base de connaissances flexibles," Université Paris XI, 1996.

[11] F. Trichet, "DSTM: un environnement de modélisation et d'opérationalisation de la démarche de résolution de problèmes d'un Système à Base de Connaissances.." Nantes: Université de Nantes, 1998.

[12] B. Wielinga, W. V. d. Velde, G. Schreiber, and H. Akkermans, "The KADS Knowledge Modelling Approach," in Proceedings of the 2nd Japanese Knowledge Acquisition for Knowledge-Based Systems Workshop, R. Mizoguchi and H. Motoda, Eds. Hitachi, Advanced Research Laboratory, Hatoyama, Saitama, Japan, 1992, pp. 23-42.

[13] J. Willamowski, "Modélisation de tâches pour la résolution de problèmes en coopération système-utilisateur," Université Joseph Fourrier Grenoble 1, 1994.

[14] M. Ikeda, K. Seta, and R. Mizoguchi, "Task Ontology Makes It Easier To Use Authoring Tools," presented at IJCAI, p. 342-351, 1997.

[15] C. Choquet, F. Danna, P. Tchounikine, and F. Trichet, "Modelling Knowledge-Based Components of a Learning 
Environment within the Task/Method Paradigm," presented at ITS'98, San Antonio (USA), 1998.

[16] M.-L. Betbeder and P. Tchounikine, "Structuring collective activities with tasks and plans," presented at IEEE International Conference on Advanced Learning Technologies (ICALT'2003), 2003.

[17] G. Paquette and P.Tchounikine, "Contribution à l'ingénierie des systèmes conseillers: une approche méthodologique fondée sur l'analyse du modèle de la tâche," Revue Sciences et Techniques Educatives 9 (3-4), pp. 157-184, 2002.

[18] A. Kobsa and W. Wahlster, User Models in Dialog Systems: Springer Verlag, 1989. 